\title{
Kulturelle Lehrerbildung - Professionalität im Horizont der Globalisierung
}

\begin{abstract}
Zusammenfassung
Kulturelle Lehrerbildung im Horizont von Globalisierung steht vor besonderen Professionsherausforderungen. Dieser Beitrag widmet sich einer theoretischen und empirischen Konkretisierung dieser räumlichen, sachlichen, zeitlichen und sozialen Herausforderungen. Die empirischen Untersuchungen zeigen dabei Momente eines möglichen Handlungsbedarfs auf, der sich im Kontext des gegenwärtigen Lehrangebots und handlungsleitender Orientierungen von Lehramtsstudierenden identifizieren lässt. Im Zuge weiterführender Analysen können Ansatzpunkte für zukunftsorientierte Neujustierungen herausgearbeitet werden.
\end{abstract}

Schlüsselworte: Kulturelle Lehrerbildung, Professionalität, Globalisierung, Differenz, Fachdidaktik

\begin{abstract}
In a globalised world, cultural education poses specific challenges for teacher education and professionalisation. This article introduces a theoretical and empirical concretisation of these spatial, factual, temporal and social challenges. The empirical investigations indicate aspects of a possible need for action concerning the current curricula of teacher education as well as the orientations guiding students in teacher training. In the future, further analyses may facilitate readjustments of these constitutive aspects of teachers' professionalisation.
\end{abstract}

Keywords: Cultural teacher training, professionalism, globalisation, subject didactics

Längst stellt die beschleunigte Entwicklung weltweiter Austausch- und Wechselbeziehungen das Leben des Einzelnen in einen globalen Zusammenhang. Nationalstaatliche Ordnungssysteme verlieren an Bedeutung und es lässt sich eine Pluralisierung des Sozial-Kulturellen beobachten. Damit verändern sich die Bedingungen und Kontexte für kulturelle Praxen. Zukunftsfähiges Lernen sollte es deshalb ermöglichen, sich in ein reflexives Verhältnis zur eigenen habitualisierten Kultur zu setzen, um entscheidungs-, gestaltungs- und hand- lungsfähig zu werden. Lehrkräfte spielen in diesem Prozess eine bedeutende Rolle, denn im schulischen Setting gelten sie als Vermittelnde und Multiplikatoren kultureller Deutungsmuster. Dies wird bislang in der universitären Lehrerbildung in Teilbereichen explizit bearbeitet (z.B. hinsichtlich von Themen interkultureller Bildung und Erziehung), stellt in einer umfassenderen Perspektive auf kulturelle Bildung im Kontext der Lehrerbildung aber ein Desiderat dar.

Hier liegt der Ansatzpunkt für den vorliegenden Beitrag: Ziel ist es, die Ausgangslage für eine im Hinblick auf Fragen der Globalisierung kulturreflexiven Lehrerbildung theoretisch und mit ersten Forschungsergebnissen 1 empirisch fundiert zu beschreiben. Das Vorhaben rekurriert auf exemplarische Professionsherausforderungen, die sich als möglicher Überschneidungsbereich des Globalen Lernens bzw. der Bildung für nachhaltige Entwicklung und kultureller Bildung identifizieren lassen.

\section{Kulturbezogene Lehrerprofessionalität im Horizont von Globalisierung}

In den nachfolgenden Ausführungen werden mit Blick auf kulturelle Bildungsprozesse relevante Aspekte in den Sinndimensionen Globalen Lernens (Lang-Wojtasik, 2008; Scheunpflug, 2003; Stichweh, 2000; Treml, 2000) aufgegriffen. Es wird danach gefragt, welchen (Lern-) Herausforderungen Lehrkräfte angesichts von Globalisierung bzw. Globalität gegenüberstehen und welche Facetten kulturbezogener Professionalität vor diesem Hintergrund besonderes bedeutend erscheinen.

Das für diese Verhältnisbestimmung zugrundeliegende Verständnis von Kultur schließt an kulturwissenschaftliche Begriffsbestimmungen an und liegt jenseits einer Beschränkung von Kultur auf den (hoch)kulturell-ästhetischen Sektor. Vielmehr geht es um Kultur als ein Bedeutungsgewebe verinnerlichter Ordnungen, Werte und Normensysteme (Eliot, 1949; Geertz, 2016; Reckwitz, 2004; Williams, 1989). Vor dem Hintergrund der Sinndimensionen Globalen Lernens werden im Folgenden Facetten kulturbezogener Professionalität von Lehrkräften herausgearbeitet. 


\section{Die räumliche Entgrenzung und Glokalisierung von Kultur}

Die gegenwärtigen weltgesellschaftlichen Entwicklungen spiegeln sich in der räumlichen Dimension in einem glokalen Raumbezug (Robertson, 1998, S. 208) über neue Medien und Kommunikationsformen, erweiterte Mobilitätsformen sowie weltumspannende Handels- und Warenströmen wider. Räumliche Entgrenzung und Glokalisierung sind dabei eingewoben in kulturelle Deutungsmuster, die sich in kultureller Pluralisierung und Diversität zeigen.

Lehrkräfte müssen Schülerinnen und Schüler auf diese Diversität und Glokalität vorbereiten. Mit Blick auf die Entgrenzung des Raumes und die Allgegenwärtigkeit einer Vielfalt von kulturellen Interpretationshintergründen stellt sich für Lehrkräfte die Herausforderung, Orientierung im entgrenzten Raum zu bieten ohne dabei singuläre kulturelle Deutungsmuster zu privilegieren oder in den Vordergrund zu stellen. Kultur kann dabei „die Funktion erfüllen, in einem entgrenzten und ,glokalen` Raum zu begrenzen und eine sinnhafte Schließung pluraler kultureller Bezüge zu ermöglichen, die für soziale Systeme anschlussfähig ist" (Lang-Wojtasik, 2008, S. 61). Voraussetzung dafür ist es, Kultur nicht als statisch und an nationalstaatliche Grenzen gebunden zu begreifen, sondern vielmehr als verhandel- und reflektierbare Bezugsgröße zu verstehen. Kultur rückt hier als „Beobachtungsstandpunkt zweiter Ordnung" (Scheunpflug, Franz \& Stadler-Altmann, 2012, S. 102) in den Mittelpunkt, in dem es um das reflexive Beobachten von Kultur geht. Kultur sowie die eigene Wahrnehmung, Beobachtung und Interpretation von kulturellen Bedeutungsgeweben wird zum Gegenstand der Reflexion. Dieses Verständnis knüpft an ein „bedeutungs-, wissens- und symbolorientiertes Kulturverständnis“" (Reckwitz, 2004, S. 7) an.

\section{Die zunehmende Kontingenz und}

\section{Beschleunigung kultureller Wissensbestände}

Damit einher geht die veränderte Bedeutung kulturellen Wissens. Die Steigerung der Vielfalt an verfügbaren Informationen ist mit einer enormen Komplexitätssteigerung verbunden. Mit dem Wachstum der Wissenspotenziale steigt die Notwendigkeit der Auswahl und Einschätzung verfügbarer kultureller Wissensbestände sowie die Kontingenz dieser Entscheidungen.

Gerade mit Blick auf kulturelle Lern- und Bildungsprozesse „stellt sich [dann] im schulischen Kontext immer das Problem einer Legitimierbarkeit von Selektionen “ (Lang-Wojtasik, 2008, S. 64). Die unidirektionale Vermittlung und Reproduktion von festgelegten kulturellen Praxen als ,Kultivierung des Kulturellen' wird im Horizont der Komplexität und Kontingenz kultureller Deutungsmuster brüchig. Damit wird es kaum noch möglich, einen klar festgelegten Kanon an Kulturgütern zu reproduzieren, ohne dabei einen Aus- bzw. Einblick in andere Perspektiven zu ermöglichen. Gleichzeitig ist Kultur aber auf Performanz und kulturelle Deutungen angewiesen, die kollektive Wissensmuster bedingen, beispielsweise ist gemeinsames Singen nur möglich, wenn alle das Lied kennen. Die damit verbundene Spannung produktiv zu gestalten, wird zu einer bedeutsamen Dimension von Lehrerprofessionalität. In der Gleichzeitigkeit von Glokalität und der Beschleunigung von Kommunikation entstehen vielfältige neue Formen von Kulturausprägungen, die sich in vielfältigen
Jugendkulturen äußern und für die hochkulturelle Prägung der Schule eine weitere Herausforderung darstellen. Der soziale Wandel vollzieht sich heute deutlich schneller als die Zeitspanne eines Generationenwechsels (Scheunpflug \& Hirsch, 2000, S. 5).

\section{Individualisierung und Pluralisierung kultureller Lebenswelten}

Einhergehend mit diesen Entwicklungen verliert Kultur als Konstrukt in sich homogener und nach außen hin abgrenzbarer Gemeinschaften, welches auf Unterschiede zwischen Menschen, z.B. in Bezug auf Traditionen, Einstellungen, Lebensweisen oder Sprachen, aufbaut und damit dem Kugelmodel von Herder (1772/2010) folgt, dramatisch an Bedeutung. Kultur und Nation können nicht mehr als deckungsgleich begriffen werden. Vielmehr erhalten Netzwerkstrukturen sowie hybride und transkulturelle Bedeutungsgeflechte neue Deutungskraft.

Als Multiplikatoren kultureller Deutungsmuster stellt sich für Lehrkräfte die besondere Herausforderung, den Umgang mit Heterogenität und Vielfalt fortwährend zu reflektieren und die Vorstellung von Kultur als homogenes - an nationale Grenzen gebundenes - Konstrukt zu hinterfragen und zu dekonstruieren. Insgesamt wird in allen Sinndimensionen des Globalen Lernens eine Komplexitätssteigerung von Bedeutungsverflechtungen sichtbar, angesichts derer kulturelle Handlungsfähigkeit mit gesteigerten Anforderungen für den Umgang mit Mehrdeutigkeiten, Wertpluralismen und Ordnungsbildungen verbunden ist. Für einen Unterricht, in dem Kultur in diesen komplexen Bedeutungsfacetten aufgegriffen wird, sollten Lehrkräfte sich selbst umfassend mit der Bedeutung von kulturellen Deutungsmustern auseinandergesetzt und ihre Perspektiven der Strukturierung kultureller Traditionen reflektiert haben. Sollen Schülerinnen und Schüler für das Leben in einer globalisierten Weltgesellschaft vorbereitet werden, so müssen Lehrkräfte befähigt werden, ihnen einen Rahmen zu bieten, in dem sie sich mit der eigenen kulturellen Prägung und den eigenen habitualisierten Normen- und Wertevorstellungen im globalen Kontext selbstkritisch auseinandersetzen können.

\section{Empirische Ergebnisse zu kulturbezogener Professionalität in der Lehrerbildung}

Im Folgenden werden erste Ergebnisse der empirischen Untersuchungen zur Lehrerbildung im Hinblick auf die beschriebenen Professionalitätsanforderungen im Horizont der Globalisierung fokussiert dargestellt. Es wurden Einführungswerke der Englischdidaktik darauf hin analysiert, welches Kulturverständnis angehenden Lehrkräften angeboten wird. Zudem werden Ergebnisse einer empirischen Untersuchung zu den handlungsleitenden Orientierungen von Lehramtsstudierenden im Umgang mit Differenz und Zugehörigkeit dargestellt.

\section{Kulturverständnis in Einführungswerken der Englischdidaktik}

Im ersten empirischen Zugang stehen Kulturverständnisse im Fokus, die in der gegenwärtigen universitären Lehrerbildung diskursiv in Einführungswerken der Englischdidaktik präsent sind. Mit der zunehmenden Relevanz der englischen Sprache 
wächst die Herausforderung, Lehrende darin auszubilden, dass sie Schülerinnen und Schülern die englische Sprache und Literatur in ihrem komplexen Wissens- und Bedeutungszusammenhang zugänglich machen. Die Identifizierung des Kulturverständnisses in englischdidaktischen Konzeptionen scheint daher im Horizont des eingangs beschriebenen theoretischen Kontexts erkenntnisbringend.

\section{Zum Forschungskontext}

In dieser Teiluntersuchung geht es um Kulturverständnisse in Fachdidaktiken geistes- und kulturwissenschaftlicher Unterrichtsfächer. Datengrundlage dieser Teilstudie stellen sieben englischdidaktische Einführungswerke dar, die im Rahmen der Grounded Theory (Strauss \& Corbin, 1996) nach dem Prozess des Theoretical Sampling (für den Projektzusammenhang siehe Franken, Lindner \& Vogt, 2019) als relevante Grundlagenliteratur der gegenwärtigen universitären Lehrerausbildung identifiziert wurden (dies sind Böttger, 2005; Doff \& Klippel, 2007; Gehring, 201033 Haß, 2006²; Thaler, 2012; Timm, 1998 und Volkmann, 2010).

Um Zugang zum (re-)präsentierten Kulturverständnis in den Einführungswerken zu erhalten, wird im Horizont einer Diskursanalyse nach „wiederkehrenden Mustern und Abweichungen von Aussagepraktiken" (Keller, 2011) mit einem inhaltsanalytischen Fokus gesucht, die sich hinsichtlich kulturund bildungstheoretischer Bedeutungszuschreibungen in der Englischdidaktik identifizieren lassen. Für die Auswertung der Daten ist die Orientierung am oben beschriebenen Theorierahmen leitend. Vor dem Hintergrund einer zunehmenden räumlichen, sachlichen, zeitlichen und sozialen Entgrenzung wird dabei eine glokale Präsentation des Englischen als Lingua franca, ein reflexiver Umgang mit kulturellen Differenzsetzungen, eine Loslösung von nationalen Kontexten, die Repräsentanz nicht britischer und nicht amerikanischer Literatur- und Sprachformen, die Problematisierung der Dominanzstellung und Verdrängung anderer Sprachsysteme sowie transkulturelle Perspektiven erwartet.

\section{Empirische Befunde: Kultur im Diskurs der Englischdidaktik} Glokalisierung und (inter-)kulturelle Reflexivität

Die räumliche Entgrenzung des Englischen wird in den untersuchten Einführungswerken als bedeutsam herausgestellt. Im Diskurs zeichnet sich dabei ein konsensuales Bewusstsein aber zugleich auch Uneinigkeit über die Bedeutung und Relevanz einer Glokalisierungsperspektive für den Englischunterricht ab. Alle untersuchten Einführungswerke thematisieren die englische Sprache als Lingua franca und deren wachsende Bedeutung als Verkehrs- und Weltsprache, die den Englischunterricht in besonderer Weise legitimiert sowie mit Blick auf tradierte Vorstellungen der Sprach- und Kulturvermittlung herausfordert. Insgesamt ist der Umfang der Thematisierung und insbesondere die konsequente Reflexion des glokalen Bedeutungszusammenhang in den Werken different angelegt: sie reicht von eher zusammenhangslos bleibender Erwähnung (z.B. Gehring, 2010, S. 19; Haß, 2006, S. 27), der skizzenhaften Thematisierung in spezifischen Unterkapiteln (z.B. Böttger, 2005, S. 44ff.; Thaler, 2012, S. 14f.; Zydatis in Timm, 1998, S. 17f.) bis hin $\mathrm{zu}$ einem Argumentationsmodus, der eine Präsentation des
Englischen als Lingua franca in den Ausführungen konsequenter mitdenkt (z.B. Doff \& Klippel, 2007, insb. S. 11ff., 38ff.; Volkmann, 2010, insb. S. 4ff., 17ff., 142ff.). In einigen Einführungswerken werden skizzenhaft Positionierungen für und gegen eine glokale Perspektive des Englischen im Unterricht angeführt (z.B. Doff \& Klippel 2007, S. 38ff.; Thaler, 2012, S. 16f.) oder es wird explizit dagegen argumentiert (z.B. Böttger, 2005, S. 46). Teilweise bleibt eine reflexive Perspektive auf eine glokale Präsentation des Englischen im Unterricht damit im Diskurs aber auch stärker ausgeblendet (z.B. Böttger, 2005; Gehring, 2010; Haß, 2006; Timm, 1998) oder wird tendenziell widersprüchlich präsentiert. Dies impliziert auch plurale Auslegungen kulturtheoretischer Bedeutungszuschreibungen des Englischen in der Fachdidaktik.

Der englischdidaktische Diskurs zeichnet sich durch einen verstärkten Rekurs auf ein weites Kulturverständnis aus, demnach Kultur „die gesamten Lebensweisen einer Gesellschaft" (Gehring, 2010, S. 15) in den Blick nimmt, wozu auch „Alltagsbereiche und Alltagswirklichkeit, Sprach- und Kulturgewohnheiten “ (ebd.) zählen. Die unterrichtliche Auseinandersetzung mit englischer Sprache und Literatur wird damit kulturgebunden verhandelt und impliziert plurale Bedeutungsverflechtungen. Insgesamt lässt sich die Vermeidung „nationalstaatlicher" Diskurslinien identifizieren. Eine Ausnahme bildet Volkmann, der „Mentalitäten und ,Nationalcharakter““ (2010, S. 73ff.) umfassend diskutiert. Trotz sprachlicher Vermeidung werden in den diskursiven Formaten der untersuchten Einführungswerke jedoch implizite Orientierungen an einem nationalstaatlichen Charakter sichtbar. Sie zeigen sich z.B. in der übergreifenden Dominanz angloamerikanischer Bezüge in Beispielen (z.B. Ortsbezüge, Literaturbeispiele, Bedeutung von Muttersprache und native speakern), der einheitlichen Orientierung der Werke am British English, der Relevanzsetzung und Reflexion landeskundlicher Wissensaneignung (z.B. Böttger, 2005) oder verstärkten Rekursen auf das Zielkultur(en)- und Zielsprache(n)-Konzept (z.B. Doff \& Klippel, 2007; Gehring, 2010; Timm, 1998; Volkmann, 2010).

In den untersuchten Einführungswerken findet die Vermeidung eines nationalstaatlichen Argumentationsmodus durch den verstärkten Rekurs auf das Konzept des (Fremd-) Verstehens englischsprachiger "Zielkultur(en)" seinen Ausdruck (z.B. Doff \& Klippel, 2007, S. 116ff.; Gehring, 2010, S. 167ff.; Thaler, 2012, S. 271ff.; Volkmann, 2010, S. 35ff.). Die kulturtheoretischen Bezüge im Kontext des (Fremd-)Verstehens von englischsprachigen Zielkulturen implizieren dabei Differenzsetzungen zwischen „eigener und fremder Kultur“" (Böttger, 2005, S. 115). Damit ist die Annahme von ,grundsätzlich weiter existierenden Unterschieden von Kulturen" (Volkmann, 2010, S. 43) in der Fachdidaktik üblich. Unter anderem bei Rekursen auf ein Verstehen und Vertrautmachen mit kulturellen Unterschieden finden sich in den Ausführungen - gerade in unterrichtspraktischer Hinsicht - häufig Homogenitätsvorstellungen von Zielkultur(en). So betont zum Beispiel Gehring, dass Verständigungskompetenzen „von Kultur zu Kultur unterschiedlich“ (2010, S. 157) sind. Sowohl „Zielsprache“ als auch „Zielkultur" werden häufig als etwas in sich homogenes präsentiert. Dies kann auch als Hinweis dafür gesehen werden, dass trotz des Rekurses auf einen weiten Kulturbegriff, dieser implizit Beschränkungen unterworfen bleibt. 
Am deutlichsten werden kulturelle Reflexivität und der Umgang mit kulturellen Differenzsetzungen im Kontext des interkulturellen Kompetenz (Böttger, 2005, S. 114ff.; Thaler, 2012, S. 271ff.; Volkmann, 2010, S. 17ff., 157ff.) und des interkulturellen Lernerwerbs (Doff \& Klippel, 2007, S. 116ff.; Gehring, 2010, S. 167ff.; Haß, 2006, S. 180ff.; Timm, 1998, S. 192ff.; Volkmann, 2010, S. 45ff.) verhandelt. Interkulturelle Theoriebezüge werden zwar in allen analysierten Einführungswerken in Unterkapiteln dargestellt, zugleich werden aber Abweichungen im Umfang der Thematisierung (z.B. stärkere Marginalisierung in Böttger, 2005; Haß, 2006; Thaler, 2012) sowie der In-Beziehung-Setzung zu hör- und sprachbezogenen Kompetenzen sichtbar. Das Interkulturalitätskonzept wird als kulturtheoretische Weiterentwicklung des Landeskundekonzepts präsentiert (Böttger, 2005; Doff \& Klippel, 2007; Haß, 2006; Volkmann, 2010; sowie indirekt Gehring, 2010; Thaler, 2012; Timm, 1998) und kann als zentrales kulturtheoretische Moment der gegenwärtigen Fachdidaktik identifiziert werden, in dem die Loslösung nationalstaatlicher Argumentationslinien und die Bedeutung kultureller Reflexivität im Diskurs kumuliert. Mit den Rekursen auf interkulturelle Kompetenzen wird die Bedeutung einer stärker dezentralisierenden Sensibilisierung für die eigene und fremde Kultur sowie auf einen respektvollen Umgang mit sprachlichen und kulturellen Unterschieden als Unterrichtsziel Bezug genommen (z.B. Doff \& Klippel, 2007, S. 117ff.; Haß, 2006, S. 182ff.; Thaler, 2012, S. 272f.; Volkmann, 2010, S. 165ff.). In diesem Zusammenhang wird in den untersuchten Einführungswerken auch der Umgang mit (Hetero- und Auto-) Stereotypen verstärkt diskutiert (z.B. Böttger 2005, S. 113f.; Haß 2006, S. 182ff.; Thaler 2012, S. 273; Timm 1998, S. 188ff.; Volkmann 2010, S. 84ff.). Deren Reflexivität bleibt aber zum Teil fraglich, wenn in beispielhaften Unterrichtsaufgaben vorgeschlagen wird, zunächst nationale Stereotype von den Schülerinnen und Schülern reproduzieren zu lassen, ehe diese in einem anschließenden Schritt decodiert werden sollen (z.B. Timm, 1998, S. 192; Volkmann, 2010, S. 102ff.). Daneben sind auch Argumente ,gegen eine Überbetonung der Überwindung von Stereotypen“ (Thaler, 2012, S. 273f.) präsent, beispielsweise wenn ausgeführt wird, dass ,in Stereotypen ein Körnchen Wahrheit" (Böttger, 2005, S. 114) steckt. Insgesamt sind diskursive Formate der Differenzsetzung zwischen eigener und fremder Kultur - nicht aber Gemeinsamkeiten - dominant, die der gewünschten „Öffnung gegenüber der anderen Kultur" (Thaler, 2012, S. 183) und damit einer räumlich entgrenzten Präsentation des Englischen entgegenstehen (können).

Vor dem Hintergrund einer räumlichen entgrenzten Präsentation des Englischen, lässt sich für die untersuchten Einführungswerke ausmachen, dass die interkulturelle Perspektive primär mit Blick auf die imaginierten Schülerinnen und Schüler aufzufinden ist, währenddessen die interkulturelle Kompetenz der imaginierten Lehrenden (insb. die Reflexion der eigenen Kulturgeprägtheit) eher eine diskursive Leerstelle bleibt.

\section{Kommunikationsorientierung und Kultiviertheitsperspektive}

Im untersuchten Diskurs findet die Kontingenz und Beschleunigung kultureller Wissensbestände Ausdruck in der Thematisierung der größer werdenden Relevanz der englischen Sprache als globales Verständigungsmittel und dem damit verbundenen
Bewusstsein, tradierte Vorstellungen des Englischunterrichts „aufzuweichen“ bzw. kritisch zu reflektieren. Dazu wird eine stärker an Alltagskommunikation orientierte Justierung von Vermittlungszielen, Unterrichtsinhalten und Lernformen konzipiert, die eine reflexive Auswahl und Präsentation kultureller Wissensbestände voraussetzt und damit die Dekodierungskompetenz der Lehrenden im besonderen Maße erforderlich macht.

Dem alltäglichen Bedeutungszuwachs des Englischen wird mit Rekurs auf die Anbahnung „kommunikativer Kompetenz" Rechnung getragen (Doff \& Klippel, 2007, S. 35ff.; Gehring, 2010, S. 85ff.; Haß, 2006, S. 181f., 67ff.; Thaler, 2012, S. 22, 107f.; Timm, 1998, S. 237f.; Volkmann, 2010, S. 18ff., 157ff.). Anstelle einer exakten Grammatikbeherrschung rückt die sprach- und hörbezogene Kommunikationsfähigkeit als Leitziel englischer Spracherwerb-Didaktik in den Fokus (z.B. stärkere Sprachpraxis, angemessener Umgang mit Sprachfehlern im Unterricht). Uneinigkeit herrscht über die Verhältnissetzung zwischen Kommunikations- und Sprachbeherrschung, die sich an der Frage eines angemessenen Mindeststandards an Sprachregelbeherrschung (z.B. Doff \& Klippel, 2007, S. 38ff.; Haß, 2006, S. 107ff.) zu entladen scheint. International English oder Global English-Perspektiven werden vereinzelt thematisiert (z.B. Doff \& Klippel, 2007, S. 39; Volkmann, 2010, S. 151ff.), sie werden für den Schulunterricht aber eher abgelehnt (z.B. Böttger, 2005, S. 46; Doff \& Klippel, 2007, S. 39) oder bleiben ausgeblendet. Es zeichnet sich hier ein gegenwärtiger Aushandlungsprozess um die Grenzen des Englischunterrichts im Diskurs ab. Tendenziell wird sich von einer pluralen Sprachpraxis im Unterricht distanziert, was lernzeitpragmatisch begründet wird: „Auf sprachliche Vielfalt kann der Unterricht angesichts der begrenzten Zeit (...) nur sehr oberflächlich vorbereiten " (Doff \& Klippel, 2007, S. 39). Dagegen lassen sich eher Argumente für eine plurale Rezeptionspraxis des Englischen auffinden (z.B. Thaler, 2012, S. 15). Vereinzelt wird vorgeschlagen, kulturell bedingte Eigenarten des Englischsprechens von Schülerinnen und Schülern (z.B. Migrationshintergrund) gezielter im Unterricht einzubeziehen, um eine globale (Hör-)Verständigung einzuüben (z.B. Doff \& Klippel, 2007, S. 44f.).

In diesem Diskussionszusammenhang wird auch sichtbar, dass Varietäten des Englischen noch keine gleichwertige Berücksichtigung und Anerkennung in der Fachdidaktik finden und eine sprachbezogene Kultiviertheitsperspektive des British English orientierend bleibt. Insgesamt wird die Dominanzstellung des British (und American) English und ihre Auflösung zu Gunsten einer entgrenzten Präsentation des Englischen in der Fachdidaktik kontrovers diskutiert (vgl. Doff \& Klippel, 2007, S. 11ff.). Volkmann weist darauf hin, dass die gegenwärtige Schulunterrichtspraxis „noch stark traditionelle Vorstellungen zur Normhaftigkeit des britischen oder amerikanischen Englisch“ (2010, S. 155) bedient. Auch in den untersuchten Werken lassen sich Hinweise dafür finden, den Unterricht „im Bereich der Grammatik an den Normen des britischen und amerikanischen Englisch“ (Doff \& Klippel, 2007, S. 14) weiterhin auszurichten (z.B. Böttger, 2005, S. 44; Haß, 2006, S. 108; Hermes in Timm, 1998, S. 225; Thaler, 2012, S. 15; Vollmer in Timm 1998, S. 241f.). In den untersuchten Einführungswerken findet die Dominanzstellung meist einleitend 
Erwähnung. Weitere englische und englisch-basierte Sprachsysteme finden in den weiteren Ausführungen dann kaum Beachtung; in illustrativen Beispielen finden sie sich gar nicht wieder. Zudem bleibt eine Problematisierung der mit der Dominanzstellung des Englischen verbundenen Verdrängung weniger relevanter kultureller Sprachsysteme im Diskurs stark ausgeklammert. Insgesamt zeichnet es sich als Argumentationsmuster ab, die Kultiviertheitsperspektive des British Englishs aus lernzeitpragmatischen Überlegungen nicht sprach- sondern eher inhaltsbezogen aufzulösen, indem globale Themen stärker zum Unterrichtsgegenstand gemacht werden (Doff \& Klippel, 2007, S. 14; Thaler, 2012, S. 15; Volkmann, 2010, S. 191ff.).

Der (alltags-)kommunikative Bedeutungszuwachs des Englischen findet z.B. auch in der Präsens handlungs- und schülerorientierter Vermittlungskonzepte Ausdruck (vgl. Böttger, 2005, S. 63ff.; Doff \& Klippel, 2007, S. 270ff.; Haß, 2006, S. 26, 230; Thaler, 2012, S. $110 f f$.$) .$

Mit Blick auf die inhaltsbezogene Präsentation kultureller Wissensbestände zeichnet sich das Kulturverständnis der Englischdidaktik-Einführungen durch eine zunehmende Loslösung vom Landeskundekonzept (Faktenwissen) hin zu einer kulturwissenschaftlichen Positionierung aus. Die Englischdidaktik rekurriert weitestgehend auf die Cultural Studies (vgl. Bach in Timm, 1998, S. 193; Böttger, 2005, S. 106ff.; Haß, 2006, S. 180; Gehring, 2010, S. 15; Volkmann, 2010, S. $45 \mathrm{ff}$. sowie stärker implizit Doff \& Klippel, 2007, S. 191; Thaler, 2012, S. 30, 171) und betont damit ein weites Verständnis von Kultur als „a whole way of life“ (Williams, 1958). Damit ist ein Kulturverständnis bedeutsam, demzufolge neben hochkulturellen vor allem alltagskulturelle Wissensbestände im Unterricht präsentiert werden sollen. In diesem Cultural-Studies-Zusammenhang wird auch die reflexive Selektion von Unterrichtsinhalten, vor allem im Kontext text-und musikbezogener Materialen verstärkt verhandelt (Doff \& Klippel, 2007, S. 128ff.; Gehring, 2010, S. 203ff.; Haß, 2006, S. 187ff.; Thaler, 2012, 251ff.; Volkmann, 2010, S. 235ff.). Hier wird weitestgehend die Bedeutung einer schülernahen Auswahl konzipiert, die aber weitestgehend inhaltlich unbestimmt bleibt. Konkrete Beispiele lassen sich kaum antreffen. Es werden aber teilweise „offene" Kriterien für die Materialauswahl angeboten, die angehende Lehrende im Entscheidungsprozess unterstützen sollen (z.B. Doff \& Klippel, 2007, S. 137f.; Haß, 2006, S. 198ff. und 208f.; Thaler, 2012, S. 67, 261; Volkmann, 2010, S. 2). Zudem wird im Diskurs auch auf die Auswahl von Jugendliteratur und -musik verwiesen, die ebenfalls inhaltlich weitestgehend unbestimmt bleibt (z.B. Doff \& Klippel, 2007, S. 149; Haß, 2006, S. 196ff., 207ff.; Thaler, 2012, S. 63ff.). Hierbei deuten sich Tendenzen eines "heimlichen“ angloamerikanischen Jugendliteraturkanons an (z.B. Böttger, 2005; Brusch \& Caspari in Timm 1998, S. 175; vgl. Volkmann, 2010, S. 254f.) sowie hinsichtlich der verstärkten Präsentation von Rock- und Popmusik der 1990er Jahre (z.B. Böttger, 2005, S. 69; Thaler, 2012, S. 65f.; Timm, 1998, S. 178ff.) generationsspezifische und angloamerikanische Orientierungen (vgl. Volkmann, 2010, S. 244f.). Insgesamt bleiben nicht-angloamerikanische Literatur- und Sprachformen in Beispielnennungen sowie konkrete Hinweise für den Einbezug jugendkultureller Themen und Formen unterpräsentiert (z.B. Hip-Hop-Musik,
Comics etc.). Obwohl der Bedeutungszuwachs des Englischen in der Fachdidaktik anerkannt wird, lassen sich zudem kaum Hinweise ausmachen, was eine zukunftsorientierte Präsentation des Englischen konkret bedeuten könnte (z.B. globale Themen).

Eine diskursive Leerstelle bleibt also, wie und in welchem Umfang die Lehrenden zukunftsorientierte und alltagsnahe Unterrichtsinhalte angemessen er- und vermitteln können. Konsens scheint, dass ein reflexiver Umgang mit kulturellen Bedeutungszuschreibungen eine zukunftsorientierte Unterrichtspraxis entscheidend flankiert. Deshalb wird auch ein flexibler und reflexiver Umgang mit dem Lehrwerk gefordert (z.B. Doff \& Klippel, 2007, S. 144ff.; Gehring, 2010, S. 201f.; Haß, 2006, S. 301ff.; Nold in Timm, 1998, S. 127ff.; Thaler, 2012, S. 80ff.; Volkmann, 2010, S. 235ff.).

\section{Pluralität und Transkulturalität}

Im fachdidaktischen Diskurs wird dem Bewusstsein um plurale englischsprachige Sozialitäten im Kontext der Bedeutung des Englischen als Lingua franca explizit Ausdruck verliehen, dennoch werden voranschreitende Individualisierungs- und Pluralisierungsprozesse, die transkulturelle Perspektiven und eine konsequente Reflexion kultureller Differenzsetzungen einschließt, weniger thematisiert und reflektiert.

Die Lehrwerke verweisen zwar auf die Bedeutung pluraler Sozialitäten, zugleich werden englischsprachige Gemeinschaften in unterrichtsbezogenen Ausführungen stark auf britische und amerikanische Gesellschaften reduziert betrachtet. Neben Großbritannien und USA, werden Australien und Kanada häufiger thematisiert. Weniger privilegierte englische Sprachgemeinschaften werden ausgeblendet (insb. afrikanische Sprachgemeinschaften). Trotz Relevanzsetzung des Englischen als Lingua franca (siehe Glokalisierung und (inter-)kulturelle Reflexivität) bleibt in der Darstellung eine Pluralität englischsprachiger Sozialitäten unterpräsentiert, womit einer tradierten Kultiviertheitperspektive des Englischen weiterhin Vorschub geleistet wird.

Mit der Orientierung an einer interkulturellen Diskurslinie, die das Aneignen von kulturspezifischen Wissen von Zielkultur(en) Differenz setzend in den Fokus stellt, bleibt eine transkulturelle Perspektivität auf englischsprachige Gemeinschaften kulturtheoretisch wie auch implizit in Ausführungen marginalisiert. Transkulturelle Theoriebezüge geraten als eine aktuelle kulturtheoretische Entwicklungslinie in nur zwei Werken der Englischdidaktik überhaupt in den Blick (Thaler, 2012, S. 275; insb. Volkmann, 2010, S. 22ff.).

In den unterrichtsbezogenen Ausführungen bleiben plurale Lebensformen und -stile innerhalb englischsprachiger Gemeinschaften (insb. Großbritannien) ausgeblendet. Implizite Bezüge lassen sich am ehesten im Kontext des Kulturverständnisses der imaginierten Schülerinnen und Schüler ausfindig machen. So wird im Horizont von Individualisierung und Differenzierung (z.B. Doff \& Klippel, 2007, S. 231ff.; Gehring, 2010, S. 238; Haß, 2006, S. 306ff.; Thaler, 2012, S. 129ff.) die Heterogenität der Schülerinnen und Schüler mit Blick auf eine angemessene Unterrichtsgestaltung in den Blick gerückt. Damit rekurriert die Englischdidaktik zwar auf ein diversitätssensibles Verständnis der Lerngemeinschaften, übersieht dessen Bedeutung aber zugleich hinsichtlich der Thematisierung 
englischsprachige(r) „Zielkultur(en)“. Ferner bleiben auch hier die pluralen Bedeutungsgeflechte, in denen die imaginierten Lehrenden selbst biografisch verwoben sind, ausgeblendet.

\section{Diskussion der Ergebnisse und Perspektiven für die Englischdidaktik}

In der Untersuchung wird deutlich, dass sich spezifische kulturtheoretische Konzepte in der Englischdidaktik etabliert haben, in denen Momente eines entgrenzten Verständnisses des Englischen Ausdruck finden. Zwei Werke zeichnen sich durch eine kulturtheoretische Perspektive aus (Doff \& Klippel, 2007; Volkmann, 2010), in der stetig Bezüge zu einer Globalisierungsperspektive hergestellt werden. Zu einem Großteil klammern die untersuchten Einführungswerke eine reflexive Entgrenzungsperspektive aber stärker aus. Als Muster des Diskurses zeichnet sich insgesamt ab, dass (inter-)kulturelle Kompetenzen hinter denen sprach- und hörbezogener im Thematisierungsumfang deutlich zurücktreten. Für die Englischdidaktik lässt sich daher der Bedarf einer ausgewogeneren Verhältnissetzung ausmachen.

Orientierungsgehalte englischer Sprach- und Literaturformen werden in ihrer räumlichen (z.B. Lingua franca, Loslösung nationalstaatlicher Argumentationslinie, interkulturelles Lernen, Fremdverstehen von Zielkulturen), sachlichen und zeitlichen (z.B. Kommunikationsfähigkeit, Sprach- und Rezeptionspraxis, Medieneinsatz, Authentizität von Quellen, Jugend- und Alltagsbezug) sowie in ihrer sozialen Dimension (z.B. Verweise auf Pluralität englischsprachiger Sozialitäten) durchaus mitgedacht. Es lassen sich aber auch erhebliche Differenzen in der Relevanzsetzung und der konsequenten Reflexion in den untersuchten Einführungswerken identifizieren.

So lässt sich beispielsweise das Potenzial ausmachen, Differenz setzende kulturtheoretische Perspektiven vor dem Hintergrund pluraler Bedeutungszuschreibungen umfassender zu reflektieren sowie interkulturelle Lerninhalte zu konkretisieren (z.B. stärker als querliegende Perspektive zu Unterrichtsinhalten konzipieren). Ein weiterer Potenzial lässt sich mit Blick auf implizite Orientierungen einer sprachbezogenen Kultiviertheitsperspektive im imaginierten Unterricht (z.B. Festhalten an British und American English-Orientierung, Ausblendung weiterer englischbasierter Sprachformen, keine plurale Sprachpraxis, Konsens über Verhältnissetzung von Kommunikation und Sprachbeherrschung) als auch einer inhaltsbezogenen Kultiviertheitsperspektive ausmachen (z.B. Literatur- und Musikkanon, mediale Relevanzsetzung, Umgang mit jugendkulturellen Inhalten, reflexive Unterrichtsgestaltung in Unbestimmtheit). Darüber hinaus könnten transkulturelle Perspektiven mehr Beachtung finden (z.B. Präsentation der Pluralität und Individualisierung englischsprachiger Sozialitäten). Ein blinder Fleck des Diskurses ist es auch, dass die Einführungswerke die imaginierten Lehrenden (sowie Autorschaft) hinsichtlich ihrer eigenen tradierten Kulturgebundenheit nicht umfassend mitdenken (z.B. wie Doff \& Klippel, 2007; Volkmann, 2010). Dies erscheint deshalb problematisch, weil fraglich ist, wie Lehrende Schülerinnen und Schülern ein reflexives Verhältnis zur eigenen habitualisierten Kultur in angemessener Form vermitteln können, wenn ihnen die eigene Kulturgeprägtheit nicht zugänglich ist. Die Herausforde- rung besteht darin, angehende Lehrende für eine reflexive Unterrichtspraxis umfassender vorzubereiten und ihnen Gestaltungsspielraum im Umgang mit Lehrplänen einzuräumen, damit beispielsweise zukunftsorientierte, alltagsnahe und jugendkulturelle Inhalte stärker verankert werden können.

Abschließend lässt sich festhalten, dass sich die Notwendigkeit einer kulturreflexiven Lehrerausbildung in der Englischdidaktik durchaus diskursiv niederschlägt. Wenn sich die Fachdidaktik den Herausforderungen der Globalisierung umfassend stellen möchte, dann müsste sie sich in den nächsten Jahren einer Weiterentwicklung fachdidaktischer Konzeptionen und damit verbunden Konkretisierungen für die Unterrichtsgestaltung widmen.

\section{Orientierungen angehender Lehrkräfte im Umgang mit Differenz und Zugehörigkeit}

Im Folgenden wird ein weiterer empirisch gestützter Zugang zur Professionalität von Lehramtsstudierenden im Horizont der Globalen Welt vorgestellt. In dieser Studie geht es um die Orientierungen angehender Lehrkräfte. Diese Orientierungen werden im Zuge der Professionalisierung im Studium mehr oder weniger zur Ausprägung eines professionellen Habitus transformiert (vgl. Helsper, 2018). Für die Untersuchung wurden mit Lehramtsstudierenden einem Theoretical Sampling (Glaser \& Strauss, 1967/1999; Strauss, 1994) folgend 26 Gruppendiskussionen mit insgesamt 101 Teilnehmenden durchgeführt. Die Gruppen wurden hinsichtlich des fachspezifischen Zugangs zu Kultur, ihrer kulturellen Praxen und ihrer Partizipations- bzw. Stellvertretungserfahrungen, z.B. in pädagogischen Tätigkeitsfeldern und zivilgesellschaftlichen Gruppen, ausgewählt. Die Diskussionen wurden mit der Dokumentarischen Methode (Bohnsack, 2010) ausgewertet. Mit dieser praxeologischen Perspektive werden die zugrundeliegenden, die Handlung strukturierenden Orientierungen als implizite Wissensbestände (Mannheim, 1964) rekonstruiert.

Aus dem Material lässt sich hinsichtlich einer kulturellen Bildung im Horizont der Globalisierung der Umgang mit Differenz und Zugehörigkeit als zentrales Thema herausarbeiten: Differenz(setzung) äußert sich demnach sowohl in ihrer Prozesshaftigkeit wie auch als Produktion sozialer Zugehörigkeit (vgl. Hirschauer, 2014). Es wird eine doppelte Perspektive einbezogen: Die der Konstruktion der eigenen Zugehörigkeit und die der Zuschreibung von Zugehörigkeiten Anderer, insbesondere Letztere durch Differenzsetzung.

Die rekonstruierten Orientierungsaspekte tragen insofern zur Konturierung von kultureller Lehrerprofessionalität in weltgesellschaftlicher Perspektive bei, als in der thematischen Zuspitzung auf Differenz und Zugehörigkeit handlungsleitende Orientierungen im Umgang mit Mehrdeutigkeit, Pluralisierung und Ordnungsbildung sowie für die Anbahnung eines reflexiven Zugangs zu kulturellen Prägungen wie habitualisierten Norm- und Wertvorstellungen sichtbar werden. Zugleich wird über den thematischen Fokus insofern an die herausgearbeiteten Kernelemente für Lehrerprofessionalität in weltgesellschaftlicher Perspektive angeschlossen, als dass die räumlichen, sachlichen, zeitlichen und sozialen Dimensionen auch in der Entfaltung von Orientierungsgehalten im Kontext von Differenz und Zugehörigkeit herausgearbeitet werden können. 


\section{Empirische Befunde}

Im offenen Eingangsimpuls für die Gruppendiskussionen wird nach dem Zugang zum Studium, zum Fach und den konkreten Lernsituationen gefragt. In den meisten Gruppendiskussionen wird das Thema Differenz und Zugehörigkeit durch die Studierenden selbst als bedeutsam eingebracht. Differenzsetzungen finden sich in Bezug auf Fachkulturen und Schulformen, auf Wissensgefälle und pädagogische Arbeitsformen. Sie sind zumeist mit bewertenden Anordnungen verknüpft, wodurch die sozialen Felder hierarchisch angeordnet konstruiert werden. Orientierend ist dann der Entwurf sozialer Strukturierung, die in klar umrissene Positionen hierarchisch gegliedert ist.

\section{Zugehörigkeitsräume zwischen statischen} Ausformungen und relationaler Mehrfachzugehörigkeit Die Orientierungen der Lehramtsstudierenden zeigen statische, klar begrenzte Konstruktionen von Kultur, die hinsichtlich ihres Anteils an Sozialität stiftender Bedeutungsgenerierung eindimensional strukturiert sind: sie umfassen ein tradiertes Set an Normen und Werten, das seine Kontur auch durch klare Abgrenzung zu weiteren Kulturen, die in ihrer Binnenstruktur ebenfalls homogen entworfen sind, gewinnt. Das Verhältnis verschiedener Kulturen im Raum ist dabei unterschiedlich akzentuiert, teils hierarchisch, teils parallel angeordnet. Dieser Orientierungsgehalt ist zumeist damit verbunden, dass die eigene Verwobenheit in unterschiedliche Bedeutungszusammenhänge und kulturelle Prägungen nicht zugänglich ist. Es wird die Orientierung daran sichtbar, den Bezugsraum der eigenen Sozialisation zu verfestigen. Dies zeigt sich daran, dass kognitive Grenzüberschreitungen abgelehnt werden, und der Relationierung verschiedener Bedeutungssysteme keine Dynamik oder ein Status des Fluiden zugeordnet wird. Zumeist ist diese Orientierung auch damit verbunden, dass eine interne Variablität der vertrauten Bedeutungssysteme oder ihre Verflochtenheit als Ausgangspunkt keine Relevanz entfalten. In diesen Fällen manifestiert sich der Umgang mit Differenz als Reproduktion vorgängiger Setzungen, woraus eine essentialistische Etablierung der Zugehörigkeit resultiert. Differenzen zeigen sich dann vor allem hinsichtlich der Anordnung der essentialisierten Kulturen (wie es beispielsweise im nachfolgenden Transkkriptausschnitt deutlich wird).

Im Spektrum der Orientierungen finden sich aber auch solche Gehalte, die von einer dynamischen Kulturkonzeption der Pluralität und Mehrdeutigkeit durchzogen sind. Sie sind mit der Reflexion einer relationalen Mehrfachzugehörigkeit und der Veränderung von Kultur durch kontextualisierte Variationen verknüpft. Das Ineinandergreifen verschiedener Bedeutungssysteme am konkreten Ort, die aus unterschiedlichen Bezügen resultieren, ist damit der Reflexion der Studierenden zugänglich. Habituell ist ein Umgang mit Differenzen und Zugehörigkeit, der kontingente und dynamische Anordnung einbezieht.

Bw Ja-was ich glaub ich auch interessant finde,-s fällt mir jetzt grad so ein,-in Naumburg ist doch dieses Wochenende dieses Afrikafestival; mit Kin-also

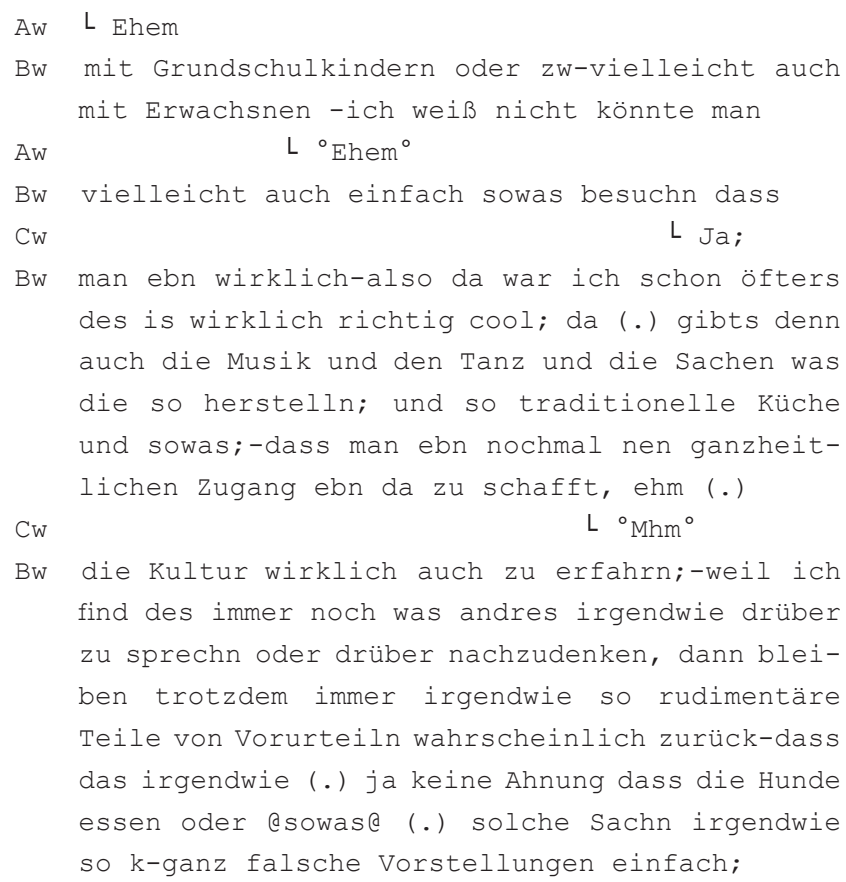

Haribo 675-688

\section{Wissen in seiner Kontextualität}

Im Orientierungshorizont der Lehramtsstudierenden lässt sich aufzeigen, dass der Umgang mit Kontingenz und der daraus resultierenden Legitimationsherausforderung von Wissen ein hohes Anforderungspotential an sie richtet. Der Schwerpunkt heteronomer Orientierungen, die das Handeln und auch das pädagogische Handeln als Erfüllung externer Normen konstruiert, leitet eine Praxis an, die sich als wiederholender Vollzug vermeintlich vorgängiger Zugehörigkeitsordnungen äußert, sowohl in Bezug auf das zu vermittelnde Wissen, dessen kontingente historische und kulturelle Einbettung nur in wenigen Fällen reflexiv zugänglich ist, als auch auf damit verbundene Entscheidungen und Differenzsetzungen. Es ist weder die Vielfalt von Interpretationsfolien im Umgang mit Bedeutungen erkennbar, noch eine Anerkennung verschiedener Wissensbestände und Interpretationshintergründe. Kennzeichnend ist eine habituelle Fremdheit zur Universität als Ort der Wissensproduktion und der Einübung einer reflexiven Haltung als Fremdheit, die Wissensinhalte in ihrer Kontextualität und Kontingenz unterschätzt.

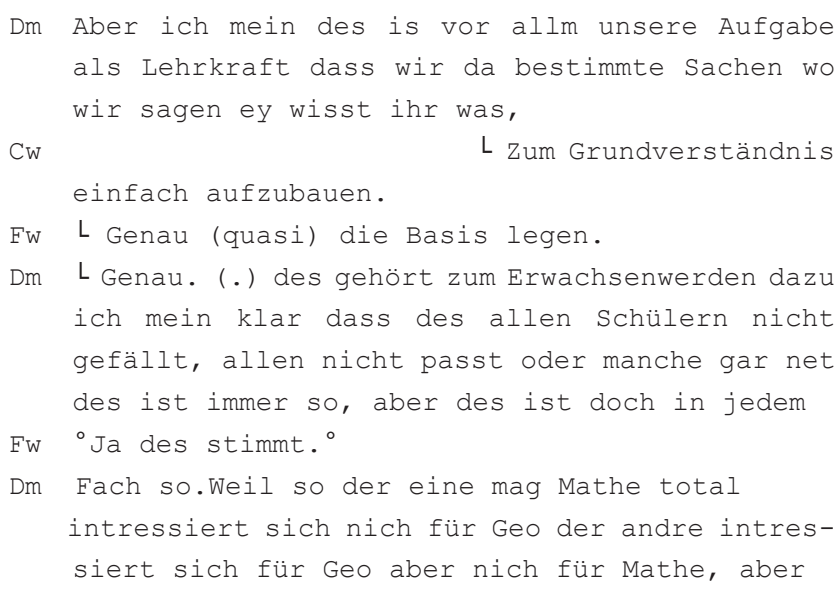




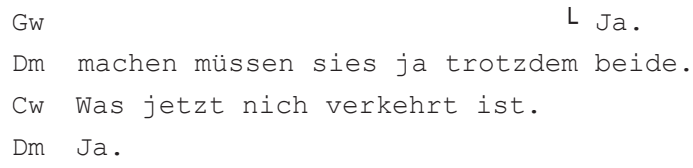

Apfelkuchen 1271-1281

Es lassen sich aber auch kontrastierende Orientierungen rekonstruieren, die mit einem reflexiven Habitus, der um die Bedingtheit und Begründungsbedarfe von Wissen und Differenzsetzungen weiß, einhergehen. Diese Orientierung ist mit einer Fragehaltung verbunden, die Veränderungen der eigenen Perspektive nicht ausschließt, und mit den Grenzen des eigenen Wissens rechnet. Diese Ausprägung der Orientierung ist mit einer Anerkennung divergenter Interpretationsfolien verbunden. Es zeigt sich dabei, dass Grenzerweiterungen und die Erfahrung von Partizipation und Selbstwirksamkeit in unterschiedlichen Kontexten Reflexionsfreiräume für den Umgang mit Kontingenz, vor allem im Blick auf Differenz und Zugehörigkeit, ermöglicht.

\section{Zugehörigkeit zwischen homogenen Kollektivsubjekten und Pluralisierungen}

In den Gruppendiskussionen werden Phänomene, die sich mit Erscheinungen und Folgen von Individualisierung und Pluralisierung in Verbindung bringen lassen, häufig thematisiert. In dieser Häufung zeigt sich, dass der Umgang mit Differenz und Zugehörigkeit für die Studierenden besondere Relevanz besitzt. Der Modus, in welchem das Thema verhandelt wird, verweist dabei auf ein hohes Irritationspotential. Es werden mehrheitlich Gehalte sichtbar, die auf eine kulturalistisch fundierte, essentialistische Zugehörigkeitsordnung verweisen. Diese zeigen sich in konsensualen Exotismen, Stereotypisierungen, Banalisierungen in den kultur-ethnischen Zuschreibungen, Diskurselementen, wie sie bereits für die räumliche Dimension relevant wurden.

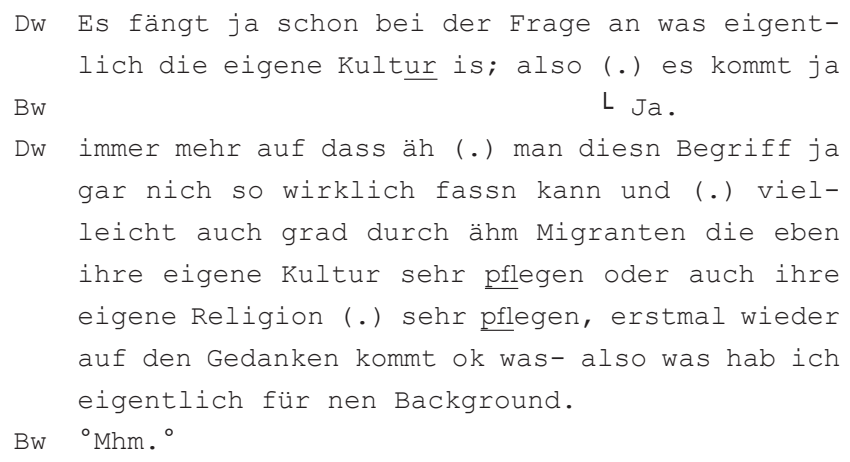

Milchreis $38-44$

Es zeigt sich eine Orientierung am Bedürfnis einer eindeutig erkennbaren Zugehörigkeit zu einem Kollektivsubjekt, zumeist als Beibehaltung und Reproduktion des in der eigenen Sozialisation erworbenen Habitus.

Differenziert werden kann der genannte Fokus in den Orientierungsgehalten in der Verknüpfung mit einer gleichzeitigen Anerkennung pluraler Werthorizonte, oder einer Ausprägung im Paradigma der Selbstverwirklichung, die in einer unterschiedslosen Beliebigkeit gegenüber Anderen mündet. Allen diesen Varianten ist gemeinsam, dass den primären sozialisierenden Instanzen eine hohe Bedeutung, der Schule als Sozialisationsinstanz dagegen eine geringe Bedeutung beigemessen wird, und pädagogisches Handeln an der Aufrechterhaltung der vorfindlichen Zugehörigkeitsordnung durch die Einsozialisation in ein Kollektivsubjekt orientiert ist. Vor diesem Hintergrund wird die Rolle des Professionellen als affirmativer Agent der institutionellen Reproduktion entworfen. Ein reflexiver Zugang zu Differenzen und Zugehörigkeit, der in einem weiteren Schritt die eigene Professionsrolle für deren Konstruktion einbezieht, ist in keiner der Varianten gegeben.

Auch für die soziale Dimension zeigen sich kontrastierende Beispiele, deren Orientierungsgehalte eine reflexive Haltung zur eigenen Sozialisation erkennen lassen. Als Modus der Weltbegegnung lässt sich Ausprobieren rekonstruieren, der sich bis zu einem gezielten Aufsuchen befremdender Erfahrungen steigert: Der Rahmen des bisherigen wird überstiegen. Differenzsetzungen und Zugehörigkeit geraten in den Fluss, Wandlung wird nicht als prekär erlebt, die Handlungsfähigkeit kann über verschiedene Kontexte hinweg aufrechterhalten werden. Differenzen und Zugehörigkeit werden plural und temporär entworfen, sowohl in Bezug auf die eigenen Bezugskontexte, wie auf deren Relationen zu einem dynamischen Außen.

\section{Diskussion der Ergebnisse}

Angesichts der hohen Herausforderungen, die für eine kulturbezogene Lehrerprofessionalität im Horizont von Globalisierung eingangs theoretisch entwickelt wurden, fällt der Blick in die Empirie ernüchternd aus. Rekonstruiert werden Orientierungsrahmen von Lehramtsstudierenden, die sich darin äußern, dass Differenzen ontologisch gerahmt werden, und die Konstruktion von Zugehörigkeit naturalisierend und essentialisierend ausfällt. Dieser Habitus lässt sich mit einem reflexiven und geläufigen Umgang mit hybriden Bedeutungschiffren, Netzwerkstrukturen, und einer Ordnungsbildung im Wertepluralismus kaum in Einklang bringen und zeigt die Grenzen der Handlungsfähigkeit der angehenden Lehrkräfte. An den Orientierungsgehalten im Blick auf Differenz und Zugehörigkeit zeigt sich, dass die Strukturierung der eigenen kulturellen Einbettung kaum reflexiv zugänglich ist, die eigenen habitualisierten Norm- und Wertvorstellungen eben gerade nicht, wie es für den globalen Kontext zwingend notwendig ist, einer kritischen Revision unterzogen werden. Diesem Ergebnis können aber weitere Aspekte beigefügt werden, die zumindest Anregungspotenziale für weitere Überlegungen zur Profilierung und Zukunftsfähigkeit der Lehrerbildung liefern können. Gruppen, die die geringe Anzahl der Kontrastfälle bilden und an dynamischen Kulturkonzeptionen orientiert sind, die den fluiden und relationalen Charakter von Differenzen reflektieren und in deren Orientierungsrahmen eine ausdifferenziert entfaltete Konzeption von Mehrfachzugehörigkeit eingelassen ist, zeichnen sich dadurch aus, dass sie in unterschiedlichen und damit auch in fremden Kontexten in einer aktiven Haltung Partizipationserfahrungen aufweisen, die mit Verantwortung und Selbstwirksamkeit verknüpft sind. Die Studierenden steuern gezielt neue Situa- 
tionen und Wissenskontexte an, setzen sich immer wieder reflexiv mit der multiplen Bedeutung kultureller Gegebenheiten auseinander und beteiligen sich an ihrer Weiterentwicklung. Die aktive Haltung und die damit verbundenen Erfahrungen scheinen dazu zu führen, der Kontingenz und Komplexitätssteigerung reflexiv und produktiv begegnen zu können.

Für solche Erfahrungen könnten in der Lehrerbildung mehr Anlässe und Herausforderungen entwickelt werden, auch wenn sie als curriculare Verpflichtung, und dann in einer heteronomen Orientierung abzuarbeitenden Aufgabe, kaum die gewünschten Effekte haben dürfte. Interessant sind auch Gruppen mit Orientierungsrahmen, deren Einzelaspekte zu kognitiven Dissonanzen führen, also etwa die Orientierung an einer eindeutig kultur-essentialistischen Zugehörigkeitsordnung wie an einer unhinterfragten Normerfüllung, deren Konfliktpotenzial dann zu Tage tritt, wenn es um Fragen der Anerkennung und Förderung eines konstruktiven Zusammenlebens von Menschen unterschiedlicher Hintergründe geht. Möglicherweise führen derartige Dissonanzen zu Lernprozessen, die als kriseninduzierte, transformatorische Bildung (Koller, 2012) aufgefasst werden können. Von dort aus ist es nicht auszuschließen, dass sie Eingang in einen Professionshabitus finden, der zu einem angemesseneren Umgang mit den Herausforderungen von Globalisierung führt.

\section{Fazit}

Die Zusammenschau der Ergebnisse verdeutlicht, dass insbesondere die Reflexion der eigenen Kulturgeprägtheit einen Handlungsbedarf markiert. So werden kulturelle Dekodierungskompetenzen in den Einführungswerken primär mit Blick auf eine schülernahe Auswahl und Präsentation von Unterrichtsinhalten diskutiert, kaum aber kulturelle Tradierungen der imaginierten Lehrenden selbst. Problematisch erweist sich das, weil rekonstruiert werden konnte, dass bei Lehramtsstudierenden gerade hier enormer Reflexionsbedarf besteht. In beiden Untersuchungen dokumentiert sich, dass Kultur in der gegenwärtigen Lehrerbildung häufig als etwas Differenz setzendes zwischen Eigenem und Fremden verhandelt wird und eigene kulturelle Tradierungen nicht umfassend reflexiv zugänglich sind. Wenn die Angebotsstruktur der universitären Lehrerausbildung hierauf nicht reagiert, dann wird einer Reproduktion tradierter Kulturverständnisse von Lehramtsstudierenden systematisch Vorschub geleistet.

Eine zentrale Herausforderung liegt darin, Lehramtsstudierenden aufzuzeigen, inwiefern sie sich selbst an exklusiven Distinktionen und an ethnisch-kulturell sowie stereotypisierenden Entwürfen von Zugehörigkeit orientieren. Die Gruppendiskussionen verweisen darauf, dass Neugierverhalten, Partizipationserfahrungen, bewältigte Fremdheitserfahrungen oder auch kognitive Dissonanzen förderliche Momente eines kulturreflexiven Professionalitätsverständnisses sind, die durch ihre systematische Berücksichtigung für eine Zukunftsausrichtung einer kulturellen Lehrerbildung stehen können. Die Vorstellung, tradierte Orientierungen von Lehramtsstudierenden im Rahmen einzelner Lehrangebote an eine kulturreflexive Perspektive „anpassen“ zu können, lässt sich nicht halten. Fraglich ist deshalb, wie eine solche Einübung durch das universitäre Lehrangebot überhaupt ermöglicht werden kann. Eine stärkere transkulturelle Neujustierung des Lehramtsstudiums könnte sich sinnvoll erweisen, weil mit ihr auf die „globalen Umwälzungen - multikulturelle Gesellschaften, Pluralisierung der Lebenswelten- und formen, Tendenz zum postnationalen Weltbürgertum“ (Volkmann, 2010, S. 22) unmittelbar reagiert wird und die Notwendigkeit „ständiger Neudefinition der eigenen kulturellen Position, sogar der kulturell geprägten Identität im Austausch mit Anderen“ (ebd.) in den Fokus rückt. Damit eröffnet diese Perspektive einen stärkeren Zugang zur Reflexion der eigenen pluralen Kulturgeprägtheit. Fachdidaktiken können z.B. stärker auf eine pluralitäts- und heterogenitätssensible Präsentation von Unterrichtsinhalten sowie einen reflexiven Umgang mit Differenzsetzungen, Homogenitätsvorstellungen und eigenen tradierten Kultiviertheitsperspektiven rekurrieren. Sinnvoll wäre hier auch, den (inter-)kulturellen Kompetenzerwerb stärker im Selbstverständnis zu verankern, ihn als eine querliegende Vermittlungsperspektive zu Unterrichtsinhalten zu konzipieren und sich gegenüber zukunftsorientierten, jugendkulturellen und medienbezogener Inhalten weiter zu öffnen. Insgesamt müssen angehende Lehrende im Studium darauf vorbereitet werden, dass kulturbezogene Professionalität eine konsequente forschungstheorie-und praxisbezogene Aus- und Weiterbildung voraussetzt. Wegeweisend können vermehrte Reflexionslehrangebote sein, die theoretisches Wissen und eigene Praxiserfahrungen umfassender in Beziehung setzen als auch verstärkte lehrveranstaltungs- und fächerübergreifende Diskussionszusammenhänge im Studium. Nicht zu vernachlässigen ist auch die Frage, wie Lehrende, die ihre universitäre Ausbildung bereits abgeschlossen haben, auf die gegenwärtigen Herausforderungen angemessen „nachbereitet“ werden können (z.B. Weiterbildungsstruktur).

\section{Anmerkung}

1 Die Daten stammen aus dem Projekt „KulturLeBi / Kultur in der Lehrerbildung - Grundlagen einer kulturbezogenen Professionalisierung angehender Lehrkräfte“, dankenswerter Weise unter dem Förderkennzeichen 01JK1602 im Förderschwerpunkt „Forschung zur kulturellen Bildung“ vom BMBF gefördert.

\section{Literatur}

Bohnsack, R. (2010). Dokumentarische Methode. In I. Miethe \& K. Bock (Hrsg.), Handbuch Qualitative Methoden in der Sozialen Arbeit (S. 247-258). Opladen/ Farmington Hills: Budrich.

Böttger, H. (2005). Einführung in die Didaktik der englischen Sprache. (Basiswissen Englischstudium, Bd. 1). Tönning/Lübeck/Marburg: Der andere Verlag.

Eliot, T. S. (1949). Beiträge zum Begriff der Kultur. Berlin: Suhrkamp.

Franken, L.; Lindner, K. \& Vogt, S. (2019): Methoden zur Erforschung kultureller Lehrerbildung im Diskurs. (im Erscheinen)

Geertz, C. (2016). The interpretation of cultures: Selected essays (EPub edition). New York: Basic Books.

Gehring, W. (2010). Englische Fachdidaktik. Theorien, Praxis, Forschendes Lernen. (Grundlagen der Anglistik und Amerikanistik, Bd. 20, 3. Aufl.). Berlin: Erich Schmidt.

Glaser, B. \& Strauss, A. L. (1967). The discovery of grounded theory: strategies for qualitative research. London: Weidenfeld and Nicolson.

Gnutzmann, C. \& Intemann, F. (2005). The Globalisation of English and the English Classroom. Tübingen: Narr.

Haß, F. (Hrsg.). (2006). Fachdidaktik Englisch: Tradition. Innovation. Praxis (2. Aufl.). Stuttgart: Ernst Klett Sprachen. 
Helsper, W. (2018). Lehrerhabitus. Lehrer zwischen Herkunft, Milieu und Profession. In A. Paseka et al. (Hrsg.), Ungewissheit als Herausforderung für pädagogisches Handeln (S. 105-140). Wiesbaden: Springer. doi.org/10.1007/978-3-658-171025_6

Herder, J. G. (1772/2010). Abhandlung über den Ursprung der Sprache. In R. Borgards (Hrsg.), Texte zur Kulturtheorie und Kulturwissenschaft. (S. 44-57). Stuttgart: Reclam.

Hirschauer, S. (2014). Un/doing Differences. Die Kontingenz sozialer Zugehörigkeiten. Zeitschrift für Soziologie, 43(3), 170-191. doi.org/10.1515/zfsoz-2014-0302

Keller, R. (2011). Diskursforschung. Eine Einführung für SozialwissenschaftlerInnen (Qualitative Sozialforschung, Bd. 14, 4. Auf.). Wiesbaden: VS.

KMK (2004). Bildungsstandards für die erste Fremdsprache (Englisch/Französisch) für den Mittleren Schulabschluss. München: Wolters Kluwer.

Koller, C. (2012). Bildung anders denken. Einführung in die Theorie transformatorischer Bildungsprozesse. Stuttgart: Kohlhammer. doi.org/10.1007/978-3-53192085-6

Lang-Wojtasik, G. (2008). Schule in der Weltgesellschaft: Herausforderungen und Perspektiven einer Schultheorie jenseits der Moderne. Weinheim: Juventa.

Luhmann, N. (1984). Soziale Systeme: Grundriß einer allgemeinen Theorie. Frankfurt a. M.: Suhrkamp.

Mannheim, K. (1964). Wissenssoziologie: Auswahl aus dem Werk. Berlin: Luchterhand.

Reckwitz, A. (2004). Kulturbegriff. In F. Jaeger \& J. Rüsen (Hrsg.), Handbuch der Kulturwissenschaften (S. 1-20). Stuttgart: Metzler. doi.org/10.1007/978-3-47605012-0_1

Robertson, R. (1998). Glokalisierung, Homogenität und Heterogenität in Raum und Zeit. In U. Beck (Hrsg.), Perspektiven der Weltgesellschaft. (S. 192-220). Frankfurt a. M.: Suhrkamp.

Scheunpflug, A. (2003). Globalisierung als Bildungsherausforderung. In J. Beillerot \& C. Wulf (Hrsg.), Erziehungswissenschaftliche Zeitdiagnosen: Deutschland und Frankreich. (European Studies in Education, Bd. 20, S. 262-278). Münster: Waxmann

Scheunpflug, A., Franz, J. \& Stadler-Altmann, U. (2012). Zur „Kultur“ in pädagogischen Zusammenhängen. In T. Fink (Hrsg.), Kulturelle Bildung: Die Kunst, über kulturelle Bildung zu forschen. (S. 99-109). München: kopaed.

Scheunpflug, A. \& Hirsch, K. (Hrsg.). (2000). Globalisierung als Herausforderung für die Pädagogik. Frankfurt a. M.: IKO.

Stichweh, R. (2000). Die Weltgesellschaft: Soziologische Analysen. Frankfurt a. M.: Suhrkamp.
Strauss, A. L. (1994). Grundlagen qualitativer Sozialforschung. München: Fink. Thaler, E. (2012). Englisch unterrichten. Grundlagen. Kompetenzen. Methoden. Berlin: Cornelsen.

Timm, J.-P. (Hrsg.). (1998). Englisch lernen und lehren: Didaktik des Englischunterrichts. Berlin: Cornelsen.

Treml, A. K. (2000). Allgemeine Pädagogik: Grundlagen, Handlungsfelder und Perspektiven der Erziehung (Kohlhammer-Urban-Taschenbücher, Bd. 441). Stuttgart/ Berlin/Köln: Kohlhammer.

UNDP (2004). Bericht über die menschliche Entwicklung 2004. Kulturelle Freiheit in unserer Welt der Vielfalt. Berlin.

Volkmann, L. (2010). Fachdidaktik Englisch: Kultur und Sprache. Tübingen: Narr. Williams, R. (1989). Resources of hope: Culture, democracy, socialism. London: Verso.

\section{Jana Costa, M. A.}

Wissenschaftliche Mitarbeiterin im Projekt Kultur in der Lehrerbildung (KulturLeBi), Arbeits- und Forschungsschwerpunkte: Kulturbezogene Aktivitäten als Lern- und Erfahrungsraum für Professionalität von Lehrkräften; Bedeutung von Globalisierungsprozessen für die Lehr- und Lernprozesse.

\section{Claudia Kühn, M. A.}

Wissenschaftliche Mitarbeiterin im Projekt Kultur in der Lehrerbildung (KulturLebi), Arbeits- und Forschungsschwerpunkte: biographie- und diskursanalytische kulturelle/ästhetische Bildungsforschung, soziale Ungleichheit, Jugend(kultur) forschung.

\section{Dr. Susanne Timm,}

Wissenschaftliche Mitarbeiterin Projekt Kultur in der Lehrerbildung (KulturLeBi), Arbeits- und Forschungsschwerpunkte: Kultur als Reflexionsfolie von Professionalität, Internationalisierungsprozesse von Bildung und Bildungssystementwicklung.

\section{Dr. Lina Franken,}

Wissenschaftliche Mitarbeiterin Universität Hamburg, Institut für Volkskunde/ Kulturanthropologie, ehemals KulturLeBi, Arbeits- und Forschungsschwerpunkte: Bildungskulturen und -politik, Kulturelle Bildung, Immaterielles Kulturerbe.

\section{UNSERE BUCHEMPFEHLUNG}

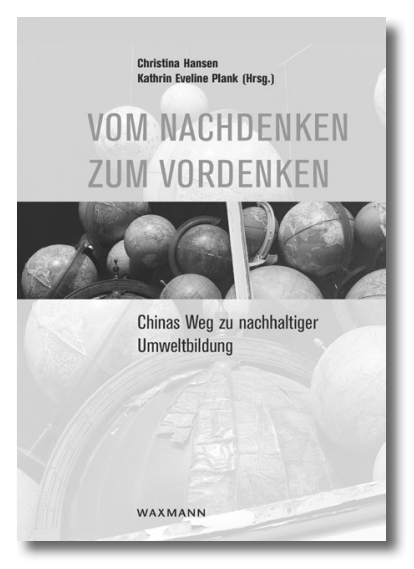

2019, 142 Seiten, br., 29,90 €, ISBN 978-3-8309-3889-7

E-Book: $26,99 €$, ISBN 978-3-8309-8889-2
Christina Hansen, Kathrin Eveline Plank (Hrsg.)

\section{Vom Nachdenken zum Vordenken Chinas Weg zu nachhaltiger Umweltbildung}

Bereits in der Lehrerbildung müssen jene Voraussetzungen geschaffen werden, mit denen angehende Lehrkräfte Kompetenzen erwerben, die sie in die Lage versetzen, Fragen nachhaltiger Entwicklung unter globaler Perspektive zu reflektieren und didaktisch aufzubereiten. Dies stellt aber eine große Herausforderung für die Lehrerbildung dar, denn es bedarf dafür mehrperspektivischer und fachübergreifender Konzepte, die auch anschlussfähig sind an unterschiedliche nationale Studienstrukturen. Schließlich gilt es, diese Konzepte für die Schule transferfähig zu machen. Wie eine solche Umsetzung auf allen Ebenen der Lehrerbildung zu realisieren ist, zeigt diese deutschchinesische Forschungsarbeit, in der ein solches Konzept entwickelt und anschließend mit 19 Schulen aus drei Provinzen in die nationale Lehrerbildung in China implementiert wurde. 